\title{
Anaerobic and Aerobic Growth of Purple Bacteria (Athiorhodaceae) in Chemically Defined Media
}

\author{
BY S. H. HUTNER \\ Haskins Laboratories, New York 17, N.Y.
}

SUMMARY: Studies on the anaerobic growth of aerobically adapted purple bacteria show that the same growth factors suffice for both aerobic and anaerobic growth, thus supporting van Niel's formulations of photosynthesis and carbon assimilation in these forms, and his thesis that the previously observed need for peptone or yeast extract could be attributed to their content of essential growth factors. Combinations of glutamate and succinate (or fumarate) were especially good promoters of growth in malate media; it is possible that their effectiveness was referable to their allowing a by-pass of the $\mathrm{CO}_{2}$ requirement. The production of molecular hydrogen by purple bacteria is discussed in relation to the reducing intensities attained in cultures.

A bottle technique for anaerobic cultures, and a flask technique for aerobic cultures, are described in detail.

Photosynthesis in purple bacteria is best observed when they are grown anaerobically (van Niel, 1944, 1949). It was therefore important to determine whether the growth factors, aneurin, nicotinic acid, biotin and $p$-aminobenzoic acid, needed for aerobic growth (Hutner, 1946), would suffice for anaerobic growth. The experiments described here indicate that although the culture media had to be modified to conform to certain special requirements imposed by anaerobic growth, the required growth factors remained the same.

The need for rigorous anaerobiosis when comparing anaerobic with aerobic growth is illustrated by Staphylococcus aureus, which requires to be given uracil only when kept strictly anaerobic (Richardson, 1936). Another cautionary example of a difference of growth-factor requirements as between aerobic and anaerobic conditions is provided by certain lactobacilli which require vitamin $\mathbf{B}_{12}$; anaerobically vitamin $\mathbf{B}_{12}$ is replaceable for them with substances which are completely inactive aerobically (Welch \& Wilson, 1949).

Investigations of this type call for the elimination of fortuitous traces of organic impurities; furthermore the confusions introduced by inapparent but growth-factor-producing microbial contamination of stock solutions have also to be eliminated. The methods here described proved adequate for the purpose and suitable for the study of photosynthetic forms: e.g. the vitamin $\mathbf{B}_{12}$ requirement of the algal flagellate Euglena gracilis (Hutner, Provasoli, Stokstad, Hoffmann, Belt, Franklin \& Jukes, 1949) was identified by these methods. A detailed account of these procedures may contribute to the reproducibility of experiments involving exceedingly active and ubiquitous growth factors.

\section{EXPERIMENTAL METHODS}

\section{Anaerobic technique}

The anaerobic bottle technique of van Niel $(1931,1936)$ served as basis. The culture vessels were ' $20 \mathrm{ml}$.' Pyrex bottles with interchangeable stoppers. The 
capacity of some bottles was $25 \mathrm{ml}$., the average being c. $24 \mathrm{ml}$.; for convenience the bottles were assumed to have a volume of $20 \mathrm{ml}$. In practice, bottles each containing $20 \mathrm{ml}$. of culture media were autoclaved with $50 \mathrm{ml}$. beakers serving as temporary covers. The glass stoppers were sterilized separately in Petri dishes. The bottles were inoculated. A reducing agent, usually $2 \%(\mathrm{w} / \mathrm{v})$ $\mathrm{Na}$ formaldehyde-sulphoxylate (Na F-S), was pipetted in to give a final concentration of $0.05 \%$. The bottles were filled to the necks with distilled water. The necks were filled with a 1:1 mixture of petrolatum and heavy paraffin oil, and sealing of the bottles was completed by replacing the covering beakers with sterile glass stoppers. The cultures were then incubated at room temperature $\left(22-80^{\circ}\right)$ under tungsten lamps. Growth was practically complete in 2 weeks with vigorous cultures; some required a month or longer. Growth was estimated visually.

Reducing agents. Na F-S was satisfactory. Its reducing intensity approached the limit set by the potential of the hydrogen electrode, as demonstrated by the ability of low concentrations $(0.02-0.04 \%)$ in culture media to reduce completely benzylviologen $\left(E_{0}^{\prime}-0.359\right.$ V.; Michaelis \& Hill, 1933) at $\mathrm{pH} 7$. That $\mathrm{Na}$ F-S induced lower potentials than thiolacetate, cysteine or ascorbate was noted by Reed \& Orr (1943). Sulphide was less satisfactory because of the narrow range between the effective reducing and toxic concentrations, and it precipitated some of the heavy metals in the medium. Na dithionite (hydrosulphite; $\mathrm{Na}_{2} \mathrm{~S}_{2} \mathrm{O}_{4} \cdot 2 \mathrm{H}_{2} \mathrm{O}$ ) proved too unstable in solution for consistent results. The following were non-toxic but lacked adequate reducing intensity: 1-thioglycerol (Evans Research and Development Corp., New York City), acetaldehyde $\mathrm{Na}$ bisulphite and $\mathrm{Na}$ benzenesulphinate. $\mathrm{Na}$ thiolacetate and mercaptoethanol were inhibitory. The oxidation-reduction system Na F-S and oxygen has not been investigated chemically. A sample of Na F-S consisting of pieces $c .1 \mathrm{~cm}$. in diameter, stored in air, kept the same reducing efficiency over a period of $3 \frac{1}{2}$ years as determined by the rough measure of anaerobic experiments.

Redox indicators. Roelofsen (1935) cultivated purple bacteria in darkness in peptone media at c. $-0.29 \mathrm{~V}$., the potential rising in light to $-0.19 \mathrm{~V}$. The cultures also produced $\mathbf{H}_{2}$ in the dark. The indigosulphonates (La Motte Chemical Products Co., Towson, Maryland) and benzylviologen (British Drug Houses Ltd.) were therefore used as redox indicators (Hewitt, 1948). The indigosulphonates were not toxic, but there were indications that benzylviologen was inhibitory at concentrations above $0 \cdot 6 \mathrm{mg} . / 100 \mathrm{ml}$. at $\mathrm{pH} \mathrm{7 \cdot 0}$. Since benzylviologen is a quaternary base resembling the bactericidal quaternary ammonium compounds, it should be less inhibitory in media more acid than those used here. The $\mathrm{Na}$ indigosulphonates were generally used at a concentration of $1.0 \mathrm{mg} . / 100 \mathrm{ml}$. and benzylviologen at $0.5 \mathrm{mg} . / 100 \mathrm{ml}$. In the reduced state benzylviologen is blue and the indigosulphonates are colourless. When media were coloured by these dyes there was definite fading after illumination for a few days, and the colour was usually completely discharged in 2-8 weeks. Aerobic or anaerobic cultures kept in the dark did not show serious fading for the duration of an experiment. This fading was rather a help; the initial full 
colour facilitated detection of insufficient anaerobiosis, and the subsequent fading simplified estimation of growth. In instances where the dyes were presumably in the colourless state rather than faded at the completion of experiments their presence was tested for, using hypochlorite for the indigosulphonates and dithionite for benzylviologen. As reactive dye invariably proved still to be present these tests were eventually discontinued. The use of high concentrations of indicator brought the advantage of a greater poising of the media; this was not noticeably advantageous when appreciable amounts of yeast extract or peptone were present, but the dyes made a significant contribution to the poising of simpler chemically defined media. In work with substrates it should be borne in mind that reduced redox indicators may serve as $\mathrm{H}$-donators (van Niel, 1931). Indigodisulphonate is readily available commercially as 'indigocarmine'.

\section{Notes}

(1) Culture bottles are available on request with a sand-blasted spot for labelling.

(2) To minimize auto-oxidation the reducing solution was prepared just before use, dispensed in deep screw-capped tubes plugged with Pyrex glass-wool (non-irritating to the respiratory tract), and the tubes quickly chilled after autoclaving. Distilled water for filling the bottles to volume was dispensed from flasks similarly fitted with glass-wool plugs, and likewise autoclaved and chilled just before use. These precautions were necessary to avoid consumption of excessive and variable amounts of reducing agent in order to reach the desired reducing intensities.

(3) When larger bottles were used the changes in fluid volume attendant upon sterilization were such that the addition of relatively large amounts of distilled water was needed. With the $20 \mathrm{ml}$. bottles water was conveniently dispensed by means of cut-down $100 \mathrm{ml}$. volumetric pipettes. These pipettes were sterilized by steam in glass tubes plugged at both ends with cotton-wool.

(4) The petrolatum-paraffin oil mixture ('vaspar') was dispensed from screwcapped bottles. For sterilization these bottles were heated in a metal desiccator for $2 \mathrm{hr}$. at $180-190^{\circ}$. To avoid entrainment of dust on cooling, the desiccator lid was sealed with Dow-Corning silicone stopcock grease. The vaspar was heated gently before pouring.

\section{Aerobic technique and general methods}

Aerobic cultures used for controls were maintained in $25 \mathrm{ml}$. Erlenmeyer flasks capped with beakers, and containing $10 \mathrm{ml}$. of medium. Pyrex flasks having a short neck (the new model) were provided with glass caps $22 \mathrm{~mm}$. high and $22 \mathrm{~mm}$. in diameter.

All stock solutions were preserved with a mixture of $(v / v) 1$ part $o$-fluorotoluene, 2 parts $n$-butyl chloride, and 1 part 1:2-dichloroethane (Hutner \& Bjerknes, 1948). This preservative volatilized on autoclaving. 0 -Fluorotoluene is obtainable in the United States from Eastman Kodak Ltd.

Media and glass stoppers were autoclaved 25-40 min. at 118-121 ${ }^{\circ}$. To avoid intake of dust on cooling, the exhaust valve of the autoclave was kept shut for at least $5 \mathrm{hr}$. after the period of sterilization. If the autoclave chamber still maintained a degree of vacuum at this time, it was brought to atmospheric pressure very gradually before opening. Petri dishes containing glass stoppers were placed in Pyrex kitchenware trays $\left(12 \frac{5}{8} \times 8 \frac{1}{8} \times 2\right.$ in. $=32 \times 20.6 \times 5 \mathrm{~cm}$.), 
as were the culture bottles and flasks. Upon removal from the autoclave the trays were covered with another inverted tray. Such double-tray containers were especially useful with the aerobic beaker-capped flask cultures. A tray of flasks could be moved about with little risk of contamination, and the double trays containing bottles or flasks could be stacked, conserving space, and, the units being transparent, conserving light. Each double-tray unit was in function the counterpart of a giant Petri dish accommodating twenty-eight $25 \mathrm{ml}$. flasks or eighteen $50 \mathrm{ml}$. flasks. For added rigidity and protection from dust the joint between the upper and lower trays was sealed with transparent cellulose tape.

\section{Culture media}

As the present work centred on the requirement for growth factors rather than on carbon or energy metabolism, it was permissible to use DL-malate throughout the experiments as metal-carrier (Hutner, 1948), hydrogen donator, and potential source of $\mathrm{CO}_{2}$. This last function depended on malate being more oxidized than cell material; therefore its complete dissimilation should lead to a net production of $\mathrm{CO}_{2}$ (Muller, 1933). Malate alone seldom permitted good anaerobic growth. Addition of glutamate, especially in combination with fumarate or succinate, allowed excellent growth; this clear-cut superiority was not evident in the aerobic controls. Good media devoid of materials of biological origin, or devoid of nitrogen except for the negligible amount contained in the growth factors, could be devised by replacing the glutamate with substrates especially suited for particular strains. Acetate and butyrate were generally useful for this purpose; glycerol was especially well utilized by

Table 1. Basal medium for identification of growth-factor requirements under anaerobic conditions

\begin{tabular}{|c|c|c|c|}
\hline & g. & & mg. \\
\hline $\mathrm{K}_{\mathbf{2}} \mathrm{HPO}_{4}$ & 0.05 & $\mathrm{Na}$ indigodisulphonate & $\mathbf{1 \cdot 0}$, or \\
\hline $\mathrm{MgSO}_{4} \cdot 7 \mathrm{H}_{2} \mathrm{O}$ & 0.025 & Benzylviologen & 0.5 \\
\hline DL-Malic acid & $\mathbf{0} \cdot \mathbf{3}$ & $\mathbf{Z n}$ & $1 \cdot 0$ \\
\hline $\mathrm{Na}_{2}$ succinate. $6 \mathrm{H}_{2} \mathrm{O}$ & $0 \cdot 4$ & $\mathbf{C a}$ & $0 \cdot 4$ \\
\hline L-Glutamic acid & $\mathbf{0 \cdot 2}$ & $\mathbf{M n}$ & $0 \cdot 2$ \\
\hline Glycerol & $0 \cdot 2$ & $\mathbf{F e}$ & $0 \cdot 1$ \\
\hline $\mathbf{K}$ acetate & $0 \cdot 1$ & $\mathbf{C u}$ & $\mathbf{0} \cdot \mathbf{1}$ \\
\hline & & Mo & 0.05 \\
\hline & & Co & 0.05 \\
\hline
\end{tabular}

Distilled water to $100 \mathrm{ml}$.

pH adjusted to $6 \cdot 8-7 \cdot 1$ with $\mathrm{KOH}$.

Na formaldehyde sulphoxylate $0.05 \mathrm{~g} . / 100 \mathrm{ml}$. added separately as a freshly autoclaved $2 \%$ solution.

Growth factors supplied when necessary as follows : aneurin $0 \cdot 1 \mathrm{mg}$., nicotinic acid $0 \cdot 1 \mathrm{mg}$., $p$-aminobenzoic acid $0.01 \mathrm{mg}$., and biotin $0.4 \mu \mathrm{g}$.

The concentrations of metals listed refer to the metal content of the salt used. These were usually the sulphates.

Rhodopseudomonas palustris. Experiments along these lines showed that all strains could use ammonium ion as principal nitrogen source. A representative medium which allowed good growth of strains of all five species studied, at a reduction level where benzylviologen developed nearly full colour and indigo- 
disulphonate was completely decolorized (the potential thus nearing that of the hydrogen electrode) is shown in Table 1.

Inoculations were carried out by suspending growth from slants of 'synthetic' agar media (made up with minimal amounts of growth factors) in the growthfactor free basal medium in aerobic flasks, and inoculating each bottle with a drop of the suspension. These dilution flasks therefore provided a check on the magnitude of carry-over effects. In other respects the procedures were those already described (Hutner, 1946).

\section{RESULTS}

The growth factor requirements for anaerobic growth appeared identical with those found for aerobic growth. Of four isolates of $\boldsymbol{R}$. capsulatus, three showed an absolute requirement for aneurin, with nicotinic acid and biotin further increasing growth; a fourth strain which grew irregularly aerobically also grew irregularly anaerobically. The four isolates tested of $\boldsymbol{R}$. gelatinosa grew in aneurin + biotin. Three isolates of $\boldsymbol{R}$. spheroides grew in aneurin + biotin + nicotinic acid. Nine out of ten isolates of $R$. palustris required $p$-aminobenzoic acid; the other strain grew poorly both aerobically and anaerobically. Seven out of nine strains of Rhodospirillum rubrum required biotin; the other two strains grew poorly.

A typical comparison of anaerobic and aerobic growth is that given for R. rubrum (Table 2). The medium was similar to that in Table 1 except that acetate was omitted. It is noteworthy that strain 6 , which is the ' $S I$ ' strain used by Gest \& Kamen $(1949 a, b)$, was one of the two strains which did not grow well in these media. The other species gave similar impressions of strain difference and of a generally faster and denser aerobic growth.

In many experiments strains grown in darkness (necessarily under aerobic conditions) showed the usual growth factor requirements.

Table 2. Comparison of anaerobic and aerobic growth of $\mathbf{R h o d o s p i r i l l u m ~ r u b r u m ~}$

(pH 7.0. Anaerobiosis was achieved by the addition of $0.04 \% \mathrm{Na}$ formaldehyde sulphoxylate. Duration of experiment: 30 days.)

$\begin{array}{llllllllll}\text { Strain: } & 1 & 2 & 5 & 6 & 7 & 10 & 15 & 17 & 20\end{array}$

Relative amounts of growth

\begin{tabular}{|c|c|c|c|c|c|c|c|c|c|}
\hline $\begin{array}{l}\text { - Biotin } \\
+ \text { Biotin }\end{array}$ & $\begin{array}{l}0 \\
+\end{array}$ & $\begin{array}{c}0 \\
++\end{array}$ & $\begin{array}{l}0 \\
+\end{array}$ & $\begin{array}{l}\mathbf{0} \\
\mathbf{0}\end{array}$ & $\frac{ \pm}{0}$ & $\begin{array}{c}0 \\
++\end{array}$ & $\begin{array}{c}0 \\
++\end{array}$ & $\stackrel{ \pm}{t+}$ & $\begin{array}{c}0 \\
++\end{array}$ \\
\hline \multicolumn{10}{|c|}{ Aerobic } \\
\hline $\begin{array}{l}\text { - Biotin } \\
+ \text { Biotin }\end{array}$ & $\begin{array}{c}0 \\
+++\end{array}$ & $\stackrel{ \pm}{+++}$ & $\begin{array}{l} \pm \\
\pm\end{array}$ & $\begin{array}{l} \pm \\
\pm\end{array}$ & $\stackrel{ \pm}{+++}$ & $\stackrel{ \pm}{+}+$ & \pm & $\stackrel{ \pm}{+++}$ & $\begin{array}{l} \pm \\
\pm\end{array}$ \\
\hline
\end{tabular}




\section{DISCUSSION}

The present findings support van Niel's thesis that the indispensability for purple bacteria of yeast extract or peptone resided in their content of essential growth factors, and that the peptone or yeast extract in his media did not significantly affect the stoichiometric relations postulated for their photosynthetic and assimilatory activities. The superior growth-promoting power of the glutamate-succinate mixtures (succinate was preferred because of its synthetic origin) pointed to a special function for these compounds. That this may be the by-passing of the $\mathrm{CO}_{2}$ requirement for initiation of growth is suggested by the work of Lwoff \& Monod (1947) who found that glutamate or succinate, preferably in combination, allowed growth of Escherichia coli in $\mathrm{CO}_{2}$ free air with a medium already containing glucose. Glutarate and asparagine were also effective, but fumarate, acetate, pyruvate, and lactate were inactive. These experiments were extended by Ajl \& Werkman (1949) to the anaerobic growth of Esch. coli and Aerobacter aerogenes: here $\alpha$-ketoglutarate, glutamate, glutamine, and oxaloacetate were the best replacements for free $\mathrm{CO}_{2}$. However, for the germination of spores of Clostridium botulinum glutamate was ineffective in replacing $\mathrm{CO}_{2}$, but a mixture of L-malate, fumarate and succinate was partially effective; oxaloacetate was completely effective, and aspartate inactive (Wynne \& Foster, 1948). In the present experiments no effort was made to deprive the cultures of $\mathrm{CO}_{2}$ nor was carbonate added; a cultivation technique permitting thorough removal of $\mathrm{CO}_{2}$ should help in elucidating the nature of the glutamate-fumarate stimulation in purple bacteria.

The isolates studied in the present investigation generally grew faster in the less reduced media. This apparent preference must be interpreted with caution as all these strains were adapted to aerobic conditions. The technique employed here allowed exposure of the inoculum to air, and hence would be ill-advised for the obligately anaerobic Rhodospirillum fulvum (Giesberger, 1947).

Certain species of purple bacteria are brown when grown anaerobically and red when grown aerobically (van Niel, 1947). This phenomenon furnished an independent way of detecting reducing conditions, but the colour shift occurred at a potential range higher than that used for the growth-factor studies.

The observations of Gest \& Kamen $(1949 a, b)$ add theoretical interest to the assessment of the likelihood that certain strains of purple bacteria may develop very low oxidation-reduction potentials. They found that a strain of $R$. rubrum produced molecular hydrogen vigorously when grown with aspartate or glutamate as nitrogen source, and with malate, fumarate or succinate as additional substrates. In agreement with the results reported here, they were able to grow their strain on a chemically defined medium containing biotin, with $\mathrm{NH}_{4} \mathrm{Cl}$ as principal nitrogen source. A determination of the potential limits for the production of hydrogen might provide an elegant method for judging the degree of anaerobiosis of cultures; should the potentials approach the region of hydrogen overvoltage some of the evolved $\mathrm{H}_{2}$ might have originated from $\mathrm{H}_{2} \mathrm{O}$ rather than from the substrate. A review of the present anaerobic bottle experiments favours the possibility that a similar evolution of $\mathbf{H}_{2}$ occurs in 
other species of purple bacteria, particularly among strains of Rhodopseudomonas palustris and $R$. capsulatus: certain strains were prone to form gas bubbles, while other strains, growing to the same extent, did not develop bubbles. Furthermore, this gas formation was not correlated with the level of oxidation of the substrate-an indication that a gas other than $\mathrm{CO}_{2}$ was being formed. The yeast extract found helpful for vigorous growth by Gest \& Kamen $(1949 b)$ appeared unnecessary when heavy metals are supplied as in the medium listed in Table 1.

This investigation was aided by a grant from the American Philosophical Society.

\section{REFERENCES}

AJx, S. J. \& Werkman, C. H. (1949). Anaerobic replacement of carbon dioxide. Proc. Soc. exp. Biol., N.Y., 70, 522.

Gest, H. \& Kamen, M. D. (1949a). Photoproduction of molecular hydrogen by Rhodospirillum rubrum. Science, 109, 558 .

Gest, H. \& KAMEN, M. D. (1949b). Studies on the metabolism of photosynthetic bacteria. IV. Photochemical production of molecular hydrogen by growing cultures of photosynthetic bacteria. J. Bact. 58, 239.

Gresberger, G. (1947). Some observations on the culture, physiology and morphology of some brown-red Rhodospirillum species. Antonie v. Leeurvenhoek J. Microbiol. Serol. 13, 135.

HewIT, L. F. (1948). Oxidation-reduction potentials in bacteriology and biochemistry, 5th ed. London: Staples Press Ltd. (Agents, London County Council.)

Hutner, S. H. (1946). Organic growth essentials of the aerobic nonsulfur photosynthetic bacteria. J. Bact. 52, 213.

Hutner, S. H. (1948). Essentiality of constituents of sea water for growth of a marine diatom. Trans. N.Y. Acad. Sci. Ser. II, 10, 136.

Hutner, S. H. \& BJerknas, ClaAra A. (1948). Volatile preservatives for culture media. Proc. Soc. exp. Biol., N.Y., 67, 281.

Hutnen, S. H., Provasoli, L., Stokstad, E. L. R., Hofrmann, C. E., Belt, M., Frankin, A. L. \& Jukes, T. H. (1949). Assay of anti-pernicious anemia factor with Euglena. Proc. Soc. exp. Biol., N.Y., 70, 118.

Lworf, A. \& Monon, J. (1947). Essai d'analyse du rôle de l'anhydride carbonique dans la croissance microbienne. Ann. Inst. Pasteur, 73, 323.

Michateis, L. \& Hul, E. S. (1933). The viologen indicators. J. gen. Physiol. 16, 859.

Muluen, F. M. (1933). On the metabolism of the purple sulphur bacteria in organic media. Arch. Mikrobiol. 4, 131.

NiEx, C. B. van (1931). On the morphology and physiology of the purple and green sulphur bacteria. Arch. Mikrobiol. 3, 1.

Niel, C. B. van (1936). On the metabolism of the Thiorhodaceae. Arch. Mikrobiol. 7, 32.

Niel, C. B. vaN (1944). The culture, general physiology, morphology, and classification of the non-sulfur purple and brown bacteria. Bact. Rev. 8, 1 .

Niel, C. B. van (1947). Studies on the pigments of the purple bacteria. III. The yellow and red pigments of Rhodopseudomonas spheroides. Antonie v. Leeurwenhoek J. Microbiol. Serol. 12, 156.

Nrel, C. B. van (1949). The comparative biochemistry of photosynthesis. In Photosynthesis in plants, p. 437, ed. J. Frank \& W. E. Loomis. Ames, Iowa: Iowa State College Press.

REED, G. B. \& ORR, J. H. (1943). Cultivation of anaerobes and oxidation-reduction potentials. J. Bact. 45, 309 . 
Richardson, G. M. (1936). The nutrition of Staphylococcus aureus. Necessity for uracil in anaerobic growth. Biochem. J. 30, 2184.

Roelofsen, P. A. (1935). On the metabolism of the purple sulphur bacteria. Proc. Acad. Sci. Amst. 37, 660.

Welch, A. D. \& WrLson, M. F. (1949). Mechanism of the growth-promoting effect of ascorbic acid on Lactobacillus leichmanii and the reduction of oxidation products of vitamin $\mathrm{B}_{12}$. Arch. Biochem. 22, 486.

Wynne, E. S. \& Foster, J. K. (1948). Physiological studies on spore germination, with special reference to Clostridium botulinum. III. Carbon dioxide and germination, with a note on carbon dioxide and aerobic spores. J. Bact. 55, 331 .

(Received 18 October 1949) 